\title{
CS Research Square \\ Nonlinear Modeling for Broadband Power Amplifiers based on Cloud Computing
}

\author{
Rina Su ( $\nabla$ srn@nbut.edu.cn ) \\ Ningbo University \\ Tai-Jun Liu \\ Ningbo University \\ Yan Ye \\ Ningbo University \\ Gao-Ming Xu \\ Ningbo University
}

Research

Keywords: nonlinear, power amplifiers ,pre-distortion, modeling

Posted Date: September 30th, 2020

DOl: https://doi.org/10.21203/rs.3.rs-79412/v1

License: (c) (i) This work is licensed under a Creative Commons Attribution 4.0 International License. Read Full License 


\section{Abstract}

This paper discusses the nonlinear dynamic behavior modeling for broadband power amplifiers based on cloud computing. The nonlinear parameters of the wideband amplifier were extracted through remote data acquisition and the analysis of the power amplifier's input and output signals. The Long Short-Term Memory neural network was used to accurately model the dynamic nonlinearity of the power amplifier. It solved the problem of limitation by time and space for the digital pre-distortion system and addressed big data remote processing for the nonlinear modeling of the broadband amplifier in $5 \mathrm{G}$ high bandwidth and high rate communication environment. By using the technology, it provides support for the efficient utilization of experimental resources and accurate nonlinear modeling of a broadband power amplifier.

\section{Introduction}

With the rapid development of modern wireless and mobile communication technology, the transmission signal goes to enter the $5 \mathrm{G}$ era. In order to support $5 \mathrm{G}$ big bandwidth and high speed, designers have to use more and more complex and efficient modulation, for example the 256QAM 8512 QAM,even1024QAM. These complex modulation and the large-scale MIMO technology make the power consumption of $5 \mathrm{G}$ base station very high. The nonlinear characteristics of the power amplifier in $5 \mathrm{G}$ base station often cause the signal regeneration or expand the spectrum outside the band. In-band transmission signal distortion results in bit-error rate performance. There is great significance to model the nonlinear behavior of a power amplifier. It is very important to reduce the efficiency of wideband power amplifiers in $5 \mathrm{G}$ base stations. The bandwidth increasing means that the power amplifier's nonlinear dynamic behavior modeling need more accuracy. It puts forward more demanding requirements for the linearity of wideband power amplifier.

On the other hand, digital pre-distortion (DPD) is one of the most promising technologies in power amplifier linearization. The digital pre-distortion technique can be used to correct the nonlinearity of a power amplifier. Digital pre-distortion systems rely heavily on the nonlinear modeling accuracy of the power amplifier. There are many proposed models including the Saleh model, Ghorbani model, a polynomial model [1-3], memory polynomial model and its extension model [2], Hammerstein model and its extended model [4], Wiener model and its extension model [5], and series-parallel mixed nonlinear model[6]. In digital pre-distortion technology, the system function which is opposite to the power amplifier's characteristic is designed by some adaptive algorithms. The input signal is preprocessed and then passed through the power amplifier, so as to cancel the nonlinear characteristic of power amplifier and realize the linearization of signal. But the traditional digital pre-distortion systems are complex based on hardware chip technology, the hardware realization of digital pre-distortion in a circuit can cause the problem of a loop delay and requires expensive equipment support, a high system cost, and high power consumption. The measure equipment is expensive, and their experimental operation is limited by the equipment. Nonlinear dynamic behavior modeling for broadband power amplifiers isn't r accurate and the remote sharing is difficult. 
Due to the increasing ability of cloud technology in data processing, cloud computing technology can be used for remote measurement and the modeling for broadband power amplifiers. Cloud technology can help realize the cloud modeling of the dynamic nonlinear behavior of power amplifiers in the environment using remote data acquisition and big data. The front-end of our system was developed based on the Django framework, and MongoDB was used for the cloud storage of the nonlinear parameter data of the power amplifier. A deep neural network Long Short-Term Memory (LSTM) was used for the nonlinear modeling of the power amplifier. The remote operation of the digital signal processing equipment effectively improved the linearization efficiency of the digital pre-distortion.

The rest of this paper is organized as follows. In Sect. 2, the DPD techniques and the nonlinear modeling characters of the power amplifier are introduced, and their performances are analyzed. In Sect. 3, a digital pre-distortion remote measurement and control system for broadband amplifiers based on cloud computing is designed, and the LSTM neural network used to model the dynamic nonlinearity of power amplifier are proposed. In Sect. 4, the test signal simulations are presented for nonlinearity model and DPD experiment. The actual experimental results compared with other methods are discussed. Finally, conclusions are provided in Sect. 5 .

\section{Background}

\subsection{Nonlinear property of power amplifier}

Power amplifier due to its circuit characteristics, the input power increases continuously, but the corresponding output does not always show a linear amplification trend. The amplifier will gradually move from the linear region to the saturated region. It will cause harmonic distortion and intermodulation distortion and affect the transmission quality of the signal.

\subsection{1 harmonic distortion}

As a nonlinear device, radio frequency (RF) power amplifier can generate parasitic signals of integral frequency of the main signal frequency when amplifying the signal. Assume that the input signal is a monophonic signal:

$$
x=A \cos (w t+\phi)
$$

The transfer function of RF power amplifier is defined as:

$$
y=f(x)=a_{1} \cdot x+a_{2} \cdot x^{2}+a_{3} \cdot x^{3}+a_{4} \cdot x^{4}+\ldots \ldots
$$

For the convenience of derivation, only the first three steps are taken and the input signal phase is assumed to be zero. At this time, the input signal is substituted in formula (2), then we can get an output expression as: 


$$
\begin{aligned}
y & =a_{1} \cdot x+a_{2} \cdot x^{2}+a_{3} \cdot x^{3} \\
& =a_{1} \cdot A \cos (w t)+a_{2} \cdot(A \cos (w t))^{2}+a_{3} \cdot(A \cos (w t))^{3} \\
& =\left(A a_{1}+a_{3} \cdot A^{3}\right) \cos (w t)+\frac{a_{2} \cdot A^{2}}{2} \cos (2 w t)+\frac{a_{3} \cdot A^{3}}{2} \cos (3 w t)
\end{aligned}
$$

From the results, the ideal monophonic signal after nonlinear transformation will get integral multiples of harmonic components $2 \mathrm{w}, 3 \mathrm{w}$ for the input signal frequency $w$. If these harmonics are not processed, the communication quality of other frequencies will be affected. But because the harmonic frequency is often far away from the main frequency, the harmonic can be filtered through a filter.

\subsection{2 intermodulation distortion}

In addition to the harmonic components, it is the intermodulation components that cause the distortion in and out of the band. The intermodulation component is different from harmonics in that its frequency component is often close to the main frequency and is difficult to be filtered by a filter.

Assume that the input dual-tone signal of the RF power amplifier is:

$$
x=A_{1} \cos \left(w_{1} t+\phi_{1}\right)+A_{2} \cos \left(w_{2} t+\phi_{2}\right)
$$

For the convenience of analysis, formula (4) can be simplified at $\left(o_{1}=0, o_{2}=0\right)$ and $A_{1}=A_{2}=1$. After simplification, the input dual-tone signal can be expressed as:

$$
x=\cos \left(w_{1} t\right)+\cos \left(w_{2} t\right)
$$

Since the input and output characteristics of the power amplifier are nonlinear, the transfer function of the RF power amplifier can be defined as:

$$
y=f(x)=a_{1} \cdot x+a_{2} \cdot x^{2}+a_{3} \cdot x^{3}+a_{4} \cdot x^{4}+\ldots \ldots
$$

Only the third order is used for expansion, and the specific expansion form is as follows: 


$$
\begin{aligned}
y= & f(x)=a_{1} \cdot x+a_{2} \cdot x^{2}+a_{3} \cdot x^{3} \\
= & a_{1} \cdot\left(\cos w_{1} t+\cos w_{2} t\right)+a_{2} \cdot\left(\cos w_{1} t+\cos w_{2} t\right)^{2}+a_{3} \cdot\left(\cos w_{1} t+\cos w_{2} t\right)^{3}+\cdots \cdots \\
= & \left(a_{1}+\frac{3}{4} a_{3}\right) \cdot \cos w_{1} t+\left(a_{1}+\frac{3}{4} a_{3}\right) \cdot \cos w_{2} t \\
& +\frac{1}{2} a_{2} \cos 2 w_{1} t+\frac{1}{2} a_{2} \cos 2 w_{2} t+\frac{1}{4} a_{3} \cos \left(3 w_{1} t\right)+\frac{1}{4} a_{3} \cos \left(3 w_{2} t\right) \\
& +a_{2} \cdot \cos \left(w_{1}+w_{2}\right) t+a_{2} \cdot \cos \left(w_{1}-w_{2}\right) t+\frac{1}{2} a_{3} \cdot \cos \left(2 w_{2}+w_{1}\right) t+\frac{1}{2} a_{3} \cdot \cos \left(2 w_{2}-w_{1}\right) t \\
& +\frac{1}{2} a_{3} \cdot \cos \left(2 w_{1}+w_{2}\right) t+\frac{1}{2} a_{3} \cdot \cos \left(2 w_{1}-w_{2}\right) t
\end{aligned}
$$

It can be seen that after the nonlinear amplification of dual-tone signals $w_{1}$ and $w_{2}$, we can obtain the first-order components, second-order components, third-order components and intermodulation components. After the signal passing through the power amplifier, in addition to the fundamental wave and frequency doubling, the mixing component is generated by the interaction between $w_{1}$ and $w_{2}$. So the ideal two-tone signal, after passing through the RF power amplifier, will derive the far-end harmonic component and the near-end intermodulation component.

\subsection{3 memory effect}

As RF power amplifier, besides nonlinear amplification of signal amplitude, it also has memory effect. In the application of narrowband signal power amplifier system, the memory effect is not obvious, but with the change of modulation mode and the continuous broadening of signal broadband, the memory performance of RF power amplifier is obvious. With the increasing requirement of communication transmission rate, the signal modulation is more and more complex, and the signal bandwidth is wider and wider. Especially for $5 \mathrm{G}$ amplifier, the wider the bandwidth, the higher the signal frequency, the more serious the memory characteristics will become. The AM/AM characteristic curves of signals with bandwidth of $25 \mathrm{MHz}, 50 \mathrm{MHz}, 75 \mathrm{MHz}$ and $100 \mathrm{MHz}$ working on RF power amplifier are respectively shown in figure 1.

It can be clearly seen that as the bandwidth gets wider and wider, the divergence degree of AM/AM characteristic graph becomes more serious and the dynamic nonlinearity becomes more obvious. Due to the strong dynamic nonlinear characteristic of high bandwidth signal, the dynamic modeling ability of the traditional model to the amplifier will be greatly reduced, so it is necessary to seek for a better model for it. Because of the better linear fitting ability of the long short-term memory neural network, we suggest using LSTM to build and simulate the dynamic nonlinear characteristics of $5 \mathrm{G}$ power amplifier.

\section{2 digital pre-distortion}

The basic principle of digital pre-distortion is to cascade a module before the input of the RF power amplifier, which is opposite to the characteristics of the RF power amplifier, so as to reverse the original 
input signal into the amplifier, and then input the reverse-distortion signal to the RF power amplifier as an input signal. Through the forward distortion of the RF power amplifier, the distortion effect of the RF power amplifier and the distortion effect of the pre-distorter can exactly cancel each other, so as to output the linearly amplified signal.

The input and output signals of the RF power amplifier are obtained from the experimental apparatus. The pre-distorter will embody the reverse characteristic of the RF power amplifier by compensating the input signal. At this time, the computer is regarded as a predistorter, and the compensated signals are converted into .wv format, which can be loaded into the signal generator. Then, the RF power amplifier is used to observe the improvement degree of the spectrum from the spectrum analyzer. After adjusting parameters, collecting data, analyze and sort out. We can get the better pre-distortion effect to correct the nonlinear characteristics of PA.

However, the current important digital pre-distortion test platform by the hardware infrastructure is expensive on the market. The data acquisition of RF power amplifier is limited which seriously restricts the digital pre-distortion technology related research.

Based on modern internet technology, relying on automatic control concept, computer cloud technology, we propose a method to design a power amplifier pre-distortion data acquisition and verification cloud platform. The platform realizes remote control of intelligent devices and remote monitoring of device status and remote acquisition of power amplifier data. LSTM network is trained through cloud big data for power amplifier modeling. Thus, it can obtain the efficient and accurate digital pre-distortion effect and the nonlinear characteristics of broadband power amplifier can be corrected, so as to improve the transmission efficiency of $5 \mathrm{G}$ signal.

\section{Proposed Method}

\section{1 digital pre-distortion system}

In this paper, we established a digital pre-distortion (DPD) remote measurement and control system for broadband amplifiers based on cloud computing. The application servers utilized included cloud computing platforms: a cloud storage server was used for the power amplifier's nonlinear feature parameter extraction, storage, and computing modeling; multiple digital pre-distorters were used for the signal compensation; a vector signal generator was used for generating signals; a high-performance spectrum analyzer was used for vector signal analysis; and power amplifiers, attenuators, couplers, and loads were used for signal processing. The digital pre-distortion structure diagram based on cloud computing is shown in Figure 2.

The application server was used to deploy the entire system using the Django architecture to complete the http requests and perform the corresponding processing. We also implemented unified centralized management, dynamic migration, and task scheduling for the pre-distortion processing of the power 
amplifier. The nonlinear amplifier was modeled through object mapping, and the template engine was connected to the parameter extraction server; a terminal was used for interaction.

The parameter extraction server for remote data transmission and acquisition was operated through the interface and the vector signal generator and power amplifier output connection. The baseband signal went to the vector signal generator and the remote acquisition amplifier output signal went through the interface calls. The system allowed for the completion of the interactions with the data from the hardware monitoring, power amplifier nonlinear characteristic parameter extraction, and storage.

The parameter extraction server used Python to call the VISA library. It realized the communication to the underlying instrument by analysis of the underlying driver through the SCPI instruction set, which helped realize the remote data transmission and collection.

We set up the test hardware environment for the power amplifier as shown in figure 3 . The test device was composed of a self-made power amplifier, Agilent vector signal generator (VSG) E4438C, spectrum analyzer E4448A (including the acquisition board card), attenuator, coupler, matching load, vector signal analysis software VSA89600, and parameter extraction server in the cloud processing module. The selected power amplifier had a rated power of $120 \mathrm{~W}$, a center frequency of $1800 \mathrm{MHz}$, and a working bandwidth of $100 \mathrm{MHz}$.

First, the signal generator, spectrum analyzer, and PC were connected to the GPIB port, and then the test signal was downloaded to the signal generator E4438C using the user interaction module software of the cloud platform. The baseband signal was converted to $1800 \mathrm{MHz}$, and then the amplifier was started. After the Radio Frequency (RF) amplifier passed through the attenuator, it passed through the coupler and was connected to the matched load. The other end of the coupler was connected to the spectrometer E4448A to display the output spectrum of the amplifier. The acquisition board card of the spectrum analyzer and the vector signal analyzer VSA89600 worked together, which converted the signal down to the baseband and captured the power amplifier's output real-time data.

Second, we evaluated the digital pre-distortion remote measurement and control system remote acquisition of various parameters, such as the sampling rate, RF power, RF frequency, and input I/Q component files. When a signal is transmitted to the power amplifier, the signal gets distorted. The input and output baseband vector signals need to be adjusted and aligned in the time delay. The cloud delay adjustment module used Numpy to adjust the delay of the input signals and output signals of the power amplifier, and then carried out the nonlinear modeling. The modeling accuracy was measured by NMSE.

Finally, the acquired pre-distortion signal was re-loaded into the signal generator E4438C to drive the rf amplifier, and the spectrum changes before and after the pre-distortion were compared using a spectrometer.

By repeating the above steps and updating the pre-distortion parameters, the pre-distortion results were gradually stabilized, and the final linearization effect of the model was obtained. 
The method of extracting the nonlinear parameters of the power amplifier mainly included capturing the original data sequence, intercepting the modeling data, delaying the adjustment, and performing the data normalization, behavior model extraction, and pre-distortion parameter extraction.

\subsection{Nonlinear Modeling for Broadband Power Amplifiers}

In this paper, LSTM neural network is used to model the dynamic nonlinearity of power amplifier. The model mainly consists of an input layer, a Batch Normalization layer, a LSTM layer, an attention layer and an output layer composed of two fully connected layers. The schematic diagram of the RF power amplifier model is shown in figure 4

Long and short term memory neural network has its unique advantages in processing long time series signals. It introduces the concept of "three gates" on the basis of cyclic neural network to avoid the disadvantages of limited memory capacity, gradient dispersion or explosion. A simple short and long term memory neuron shown in figure 5 , it has an input gate, a forgetting gate, and an output gate.

As shown in the figure, the input gate determines the weight assignment of the input matrix to obtain the status of the updated current moment:

$$
i(n)=\sigma\left(W_{\Lambda} x(n)+W_{i y} y(n-1)+b_{i}\right)
$$

The forgetting gate determines the influence factor of the state quantity at the network historical moment on the current moment, and updates the state quantity together with the current state:

$$
\begin{aligned}
& f(n)=\sigma\left(W_{f x} x(n)+W_{b j} y(n-1)+b_{f}\right) \\
& \tilde{c}(n)=g\left(W_{\alpha} x(n)+W_{c y} y(n-1)+b_{c}\right) \\
& c(n)=f(n) \cdot c(n-1)+i(n) \cdot \tilde{c}(n)
\end{aligned}
$$

The output gate determines the network output according to the updated network status:

$$
\begin{aligned}
& o(n)=\sigma\left(W_{\alpha x} x(n)+W_{o y} y(n-1)+b_{o}\right) \\
& y(n)=o(n) \cdot h(c(n))
\end{aligned}
$$

The network parameters are still solved by error back propagation algorithm.

\section{Results And Discussion}


The test signal was the 5GNR signal with a center frequency of $1800 \mathrm{MHz}$ and a sampling rate of 92.16 MSPS with a bandwidth of $100 \mathrm{MHz}$. The test input signal was input to the VSG. The output signal generated by the VSG was fed into the test amplifier, and the VSA obtained the sample data of the input and output of the amplifier. After the extraction of the parameters of the rf power amplifier's behavior model, we carried out the experiment for the power amplifier linearization based on cloud computing.

We used the following steps:

(1) the original input signal of the continuous sequence and the output signal of the power amplifier were captured by the cloud platform to establish a database and cloud storage using 20,000 data points.

(2) We took the maximum output power point of the amplifier as the interception center, intercepted a short section of data (we used 4000 data points as the training data to extract parameters from the model), and then simultaneously captured the input and pre-distortion signals of the amplifier of the same length for modeling.

(3) The original signal was fed into the pre-distorter, DAC, up-converter, RF amplifier, coupler, downconverter, $A D C$, and other devices to obtain the output sequence of the power amplifier. The interception data were delayed and adjusted so that the pre-distorter signal and the output signal of the power amplifier were at a one-to-one correspondence.

(4) We normalized the feedback data of the power amplifier. The normalized data were used to model the amplifier's behavior, and the LSTM model was used. To compare the modeling accuracy of power amplifier, LUT model, memory polynomial model, RNN model, and LSTM model were selected.

The model precision of RF power amplifiers can be measured by normalized mean square error (NMSE) . The comparison of different model is shown in Table 1. It can be seen that the accuracy of LSTM model is better than the others.

Table 1 NMSE Comparison of Different Model

\begin{tabular}{cc}
\hline \hline MODEL & NMSE \\
\hline LUT & -26.49 \\
MP & -28.49 \\
RNN & -30.84 \\
LSTM & -34.27 \\
\hline \hline
\end{tabular}

(5) We extracted the pre-distortion parameters from its inverse characteristics, which were directly updated to the pre-distorter to complete the parameter update.

(6) We repeated Steps (1)-(5) to extract and update the parameters again.

In order to verify the linearization performance effectiveness of the proposed approach, it is applied to the DPD system. For modern $5 \mathrm{G}$ communication system, to prevent the band spectrum extension is more essential than in-band spectrum correcting, so linear effect is good or bad mainly through the spectrum 
observation, through the adjacent channel power ratio (ACPR) measurement. The comparison of measured power spectrum between the proposed method with previous works is shown in figure 6

Correspondingly, the comparison of ACPR is shown in Table 2.

Table 2 ACPR Comparison of Different Model

\begin{tabular}{crr}
\hline \hline Model & \multicolumn{2}{c}{ ACPR(dBc) } \\
\cline { 2 - 3 } & Up Channel & Down Channel \\
\hline LUT & -24.32 & -28.69 \\
MP & -32.59 & -36.12 \\
RNN & -34.57 & -38.79 \\
LSTM & -39.13 & -41.38 \\
\hline \hline
\end{tabular}

By comparing the power density diagram and ACPR, it can be seen that the pre-distorter based on LSTM amplifier model has the better rectifying effect for the linearization of the power amplifier. The improvement effect for linearization is obvious compared with the traditional pre-distorter.

\section{Conclusions}

Compared with the prior method, the method proposed in this paper has the advantages of using cloud centralized management and uniform hardware system environment for data remote acquisition and training modeling, which greatly reduces the hardware cost. Nonlinear modeling of wideband PA is produced precisely by LSTM. The digital pre-distortion is realized based on the cloud platform. The remote control and sharing of instruments provide support for efficient use of experimental resources and accurate nonlinear modeling of broadband amplifier.

\section{Abbreviations}

LSTM: Long Short-Term Memory; PA: Long Short-Term Memory; ACPR: adjacent channel power ratio; NMSE: normalized mean square error; SCPI: Standard Commands For Programmable Instrumentation; RF : Radio Frequency; VSG: vector signal generator; VSA: Vector Signal Analyzer; VISA: Virtual Instrumentation Software Architecture

\section{Declarations}

\section{Acknowledgements}

The authors thank for the valuable and constructive comments from the editor and reviewers.

\section{Authors' contributions}

The work presented here was carried out in collaboration between all authors. The general idea was proposed by TJL. RNS,YY and GMX designed simulations. RNS,CC performed the experiments, analyzed 
the results. RNS wrote the paper. TJL and GMX revised the manuscript. All authors read and approved the final manuscript.

\section{Funding}

This work is supported by the National Natural Science Foundation of China under Grant 61571251, Zhejiang Provincial Public Welfare Technology Application Research Project LGG19F030007.

\section{Availability of data and materials}

Please contact the author for data requests.

\section{Competing interests}

The authors declare that they have no competing interests.

\section{Ethics approval and consent to participate}

Not applicable.

\section{Publisher's Note}

Springer Nature remains neutral with regard to jurisdictional claims in published maps and institutional affiliations.

\section{Author details}

1.Faculty of Electrical Engineering and Computer Science, No. 818 Fenghua Road, Jiangbei District, 315010Ningbo, Zhejiang Province, China

2.College of Electronic and Information Engineering, Ningbo University of Technology, No. 210 Fenghua Road, Jiangbei District, 315012Ningbo,Zhejiang Province, China

\section{References}

[1]Canavate Sanchez M C,Segneri A,Georgiadis A,et al. System performance evaluation of power amplifier behavioural models. IET Active and Passive RF DevicesS Seminar,London,2018

[2]Sappal S. Simplified memory polynomial modelling of power amplifier. 2015 International Conference and Workshop on Computing and Communication (IEMCON), Vancouver, 2015. 1-7

[3]Pan W, Liu Y, Tang Y. A predistortion algorithm based on accurately solving the reverse function of memory polynomial model. IEEE Wireless Communications Letters, 2012, 1(4):384-387

[4]Ye Y, Liu T J, Sioris J, et al. Linearization for Doherty RF power amplifiers using augmented Hammerstein dynamic nonlinear models. Journal of Microwaves, 2009,25(1):58-62 
[5]Liu T, Boumaiza S, Helaoui M, et al. Behavior modeling procedure of wideband RF transmitters exhibiting memory effects. IEEE MTT-S International Microwave Symposium Digest, Long Beach, CA, 2005

[6]Hammi O, Ghannouchi F M. Twin nonlinear two-box models for power amplifiers and transmitters exhibiting memory effects with application to digital predistortion. IEEE Microwave and Wireless Components Letters,2009, 19(8): 530-532

[7].ADEL A. M. SALEH, Frequency-Independent and Frequency-Dependent Nonlinear Models of TWT Amplifiers. IEEE Transactions on communications,vol.29, no.11,pp.1715-1720, Nov.1981

[8].G.P. White, A.G. Burr and T.Javomik, Modelling of nonlinear distortion in broadband fixed wireless access systems . Electronics letters, vol. 39, no. 8, pp. 686 -687, Apr. 2003.

[9]. Hai-Han, L., T. Shah-Jye, and L. Yen-Liang, Intermodulation distortion suppression in a full-duplex radio-on-fiber ring network. IEEE Photonics Technology Letters, 2004. 16(2): p. 602-604.

[10]. Tabatabai, F. and H.S. Al-Raweshidy. Optical predistortion feedback linearization for suppressing the nonlinearity in optical amplifier. Microwave Conference, 2008. APMC 2008. Asia-Pacific. 2008.

[11]. Yi, D., L. Zhaohui, T. Xiangqing, et al., Optical Fiber Polarization Interferometer for Performance Improvement in Radio-Over-Fiber Systems, IEEE Photonics Technology Letters, 2007. 19(16): p. 12361238.

[12]. Junghwan, M. and K. Bumman, Enhanced Hammerstein Behavioral Model for Broadband Wireless Transmitters, IEEE Transactions on Microwave Theory and Techniques, 2011. 59(4): p. 924-933.

[13]. Fernando, X.N. and A.B. Sesay, Adaptive asymmetric linearization of radio over fiber links for wireless access, IEEE Transactions on Vehicular Technology, 2002. 51(6): p. 1576-1586.

[14]. LIU Tai-jun,CHEN Hao,SU Ri-na, et al.,Neural Networks Based Nonlinear Dynamic Behavior Modelling for Broadband Power Amplifiers, Journal of microwave, vol. 36, no. 1, pp. 131 -136, Feb. 2020.

\section{Figures}




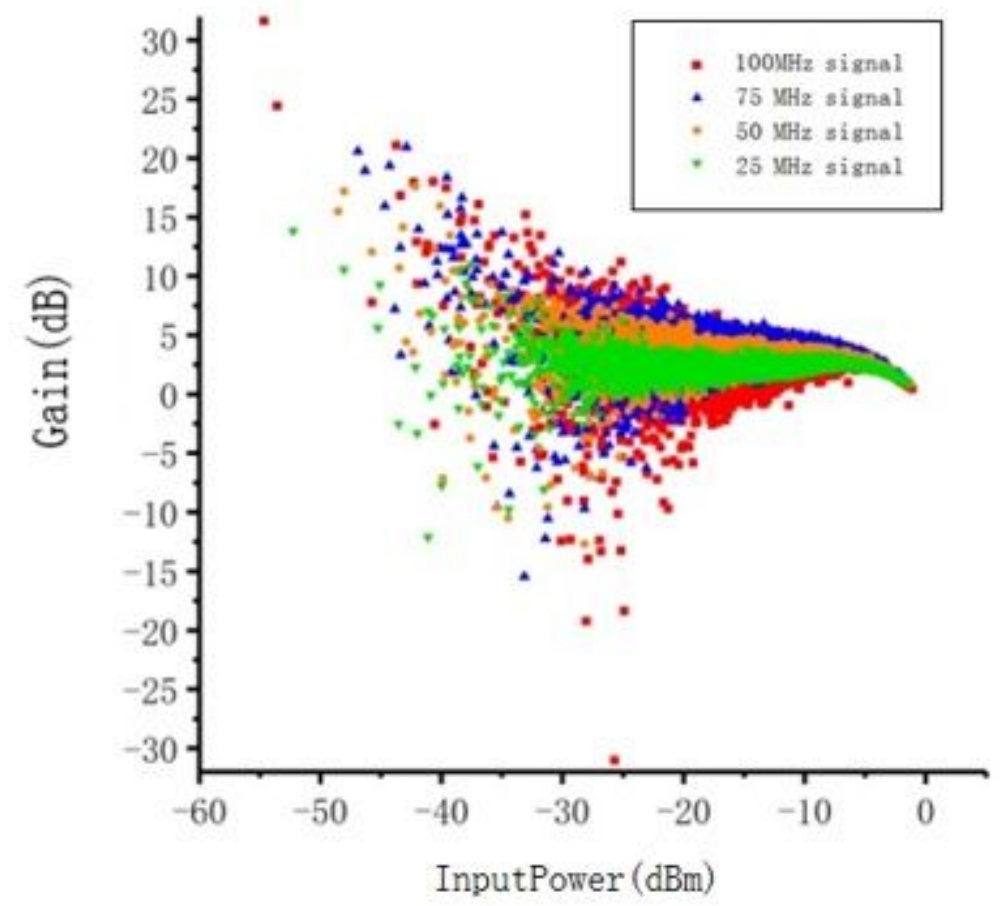

Figure 1

AM / AM picture of different bandwidth signals $(25 \backslash 50 \backslash 75 \backslash 100 \mathrm{MHz})$

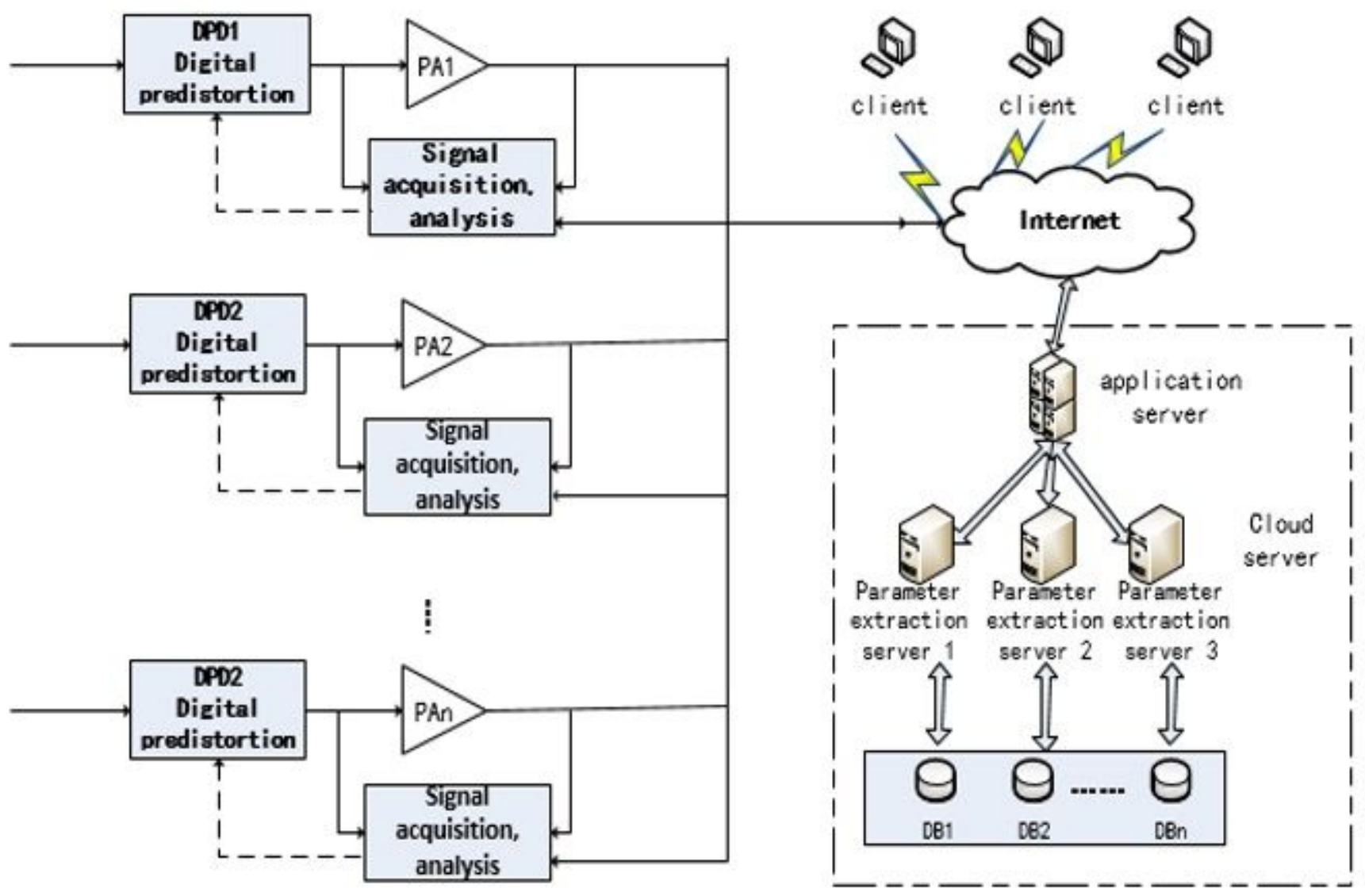


Digital pre-distortion structure diagram based on cloud computing

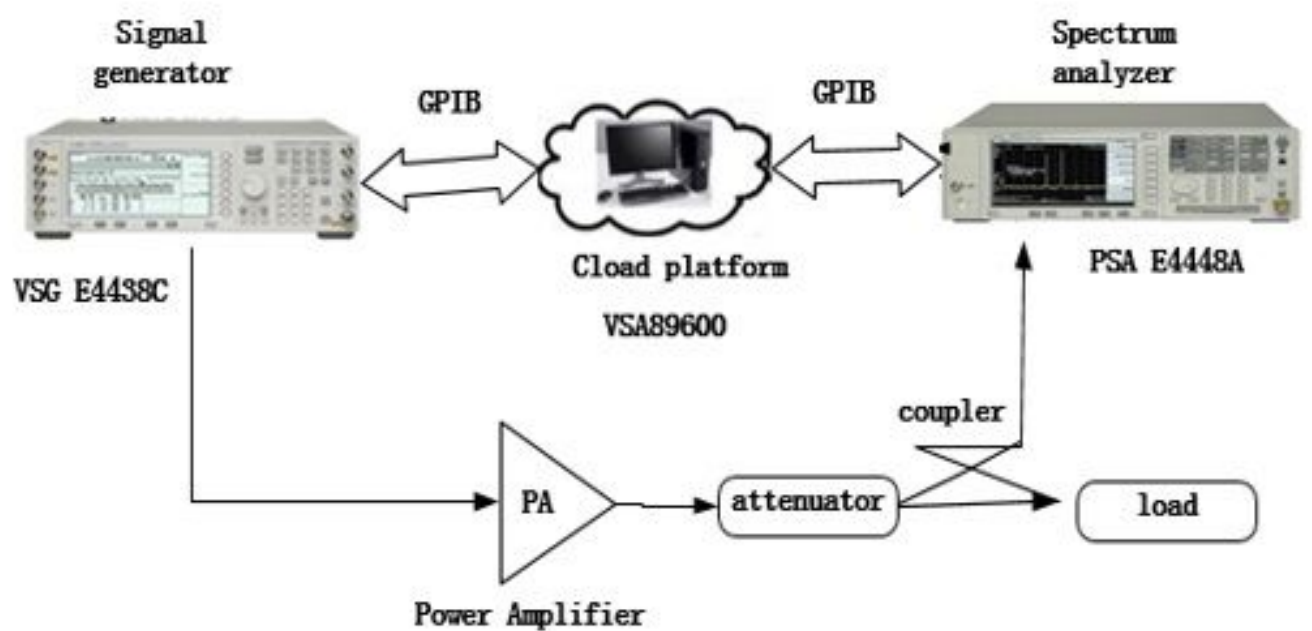

Figure 3

Digital predistortion hardware platform

$\begin{array}{llccc}\text { Input } & \text { Batch Normalization } & \text { LSTM } & \text { Attention } & \text { Output } \\ \text { layer } & & \text { layer } & \text { layer } & \text { layer }\end{array}$

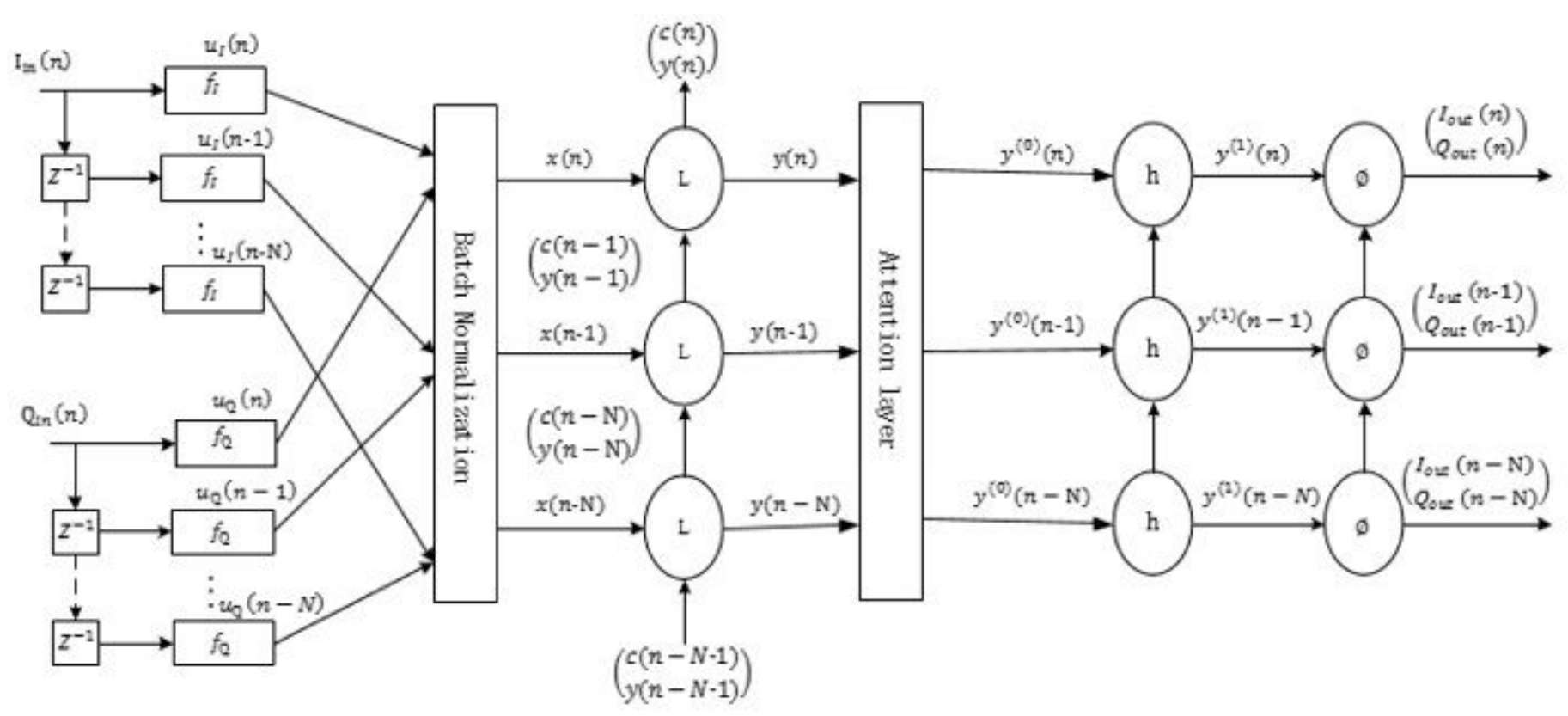

Figure 4

LSTM structure 


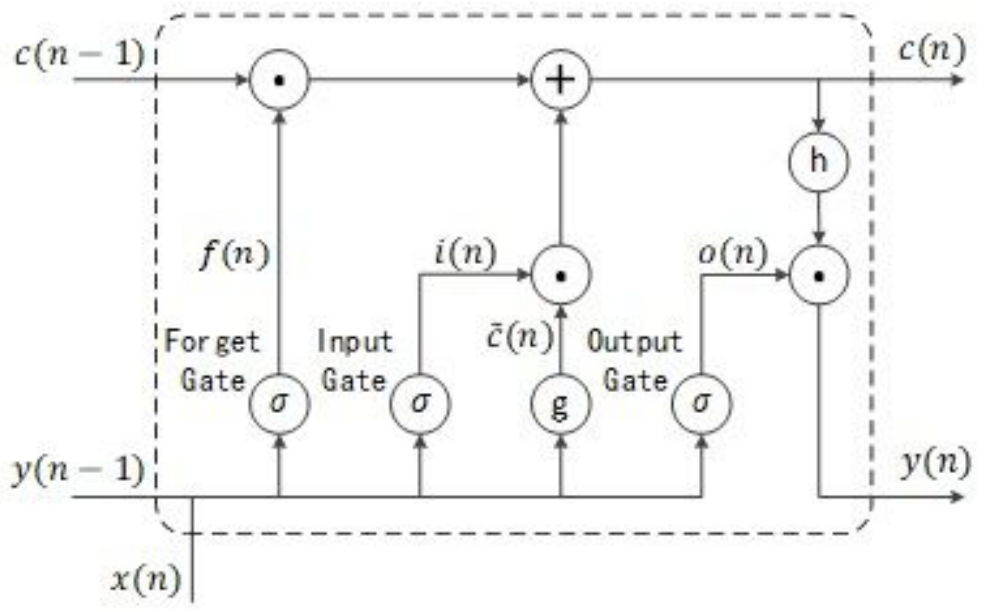

Figure 5

schematic diagram of long and short term memory neurons

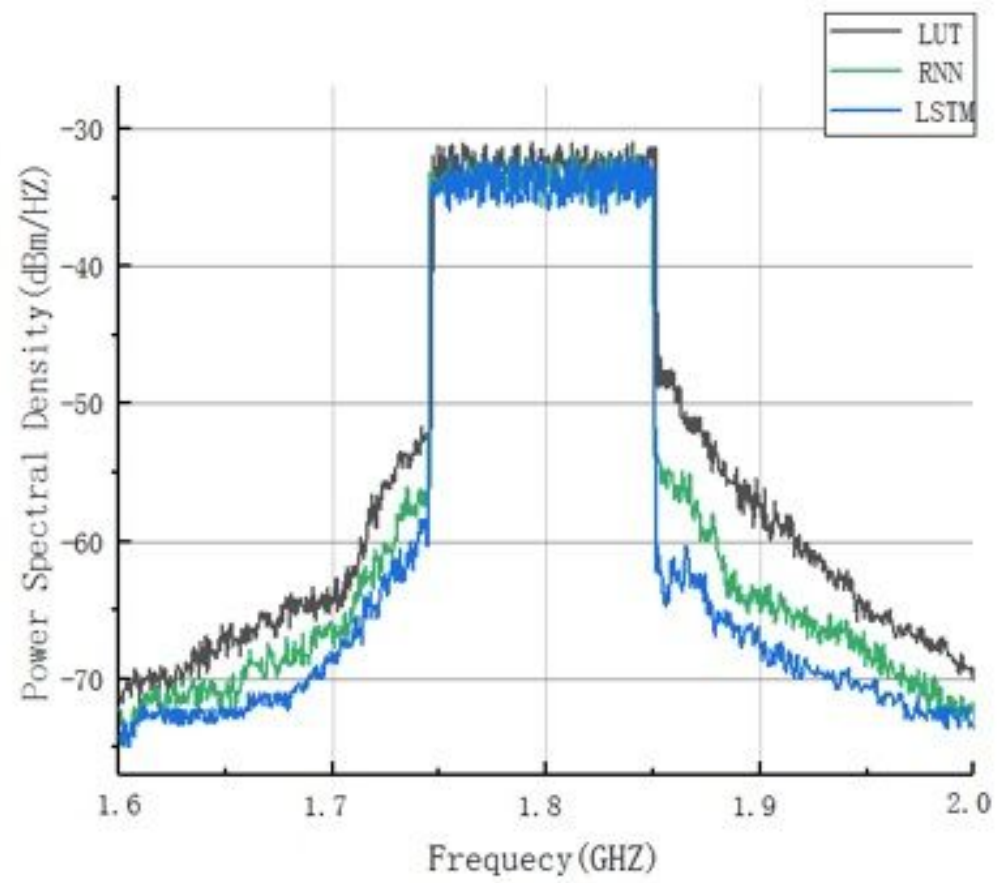

Figure 6

Power Spectral Density of Different Model 